\section{THE PROGRESS OF THE TELEGRAPH *} III.

WHENEVER the finger of scientific research points the way to mechanical applications, the creative powers of the human brain originate a multitude of inventions. Too often, however, like the rank growth of weeds which spring up to choke the produce of the soil, they surround as parasites the principles involved, and by misapplied talent, frustrate the simplicity and vigour of the original idea. By bundreds in all forms and shapes have telegraphic inventions crowded into the field ; but ninetynine out of every hundred patented inventions are not worth even the fees paid to Government. As with the multitude of steam-boiler patents, so with telegraph patents, a very limited number of the different patented inventions have survived to render any really practical aid to the everyday requirements of telegraphic transmissions by land or by sea on a large scale. All the earlier inventions, the five needle, double needle, and the single needle telegraph, Bain's chemical printer, the mechanical alphabetical printer, Morse transmitter, and others of a similar type, have long since been laid on the shelf as incompetent as regards submarine cable transmissions over extended lengths ; and a form of apparatus, more or less derived from a skilful combination of old principles and appliances, have taken their place for practical utility. These instruments, to which the descriptions in the present instance will be confined, may be classified into two distinct groups, namely, "recording" and "non-recording" instruments ; or those which mechanically record the signals on paper, and those which are read by the eye or ear, the signals afterwards being registered by hand. Before proceeding to investigate the combinations of principles employed, it is desirable to point out that these several classes of instrument have each a special department for which they are specially adapted. Thus, for submarine cable transmissions the non-recording apparatus, depending upon the correctness of the eye or the ear, must at all times be liable to error, the accuracy and precision of sight and hearing of the reader being the only voucher that the transmitter of the message has that it has been faithfully interpreted at the distant station. Mistakes under this system must therefore, of necessity, frequently arise. Instruments of the recording type are, in consequence, always to be preferred.

In all these various forms of apparatus no new principles have been discovered; they are simply successful mechanical arrangements and combinations of certain wellknown electrical laws, producing new and useful results. These fundamental principles may generally be described as follows :-

When a length of insulated wire is wound round a

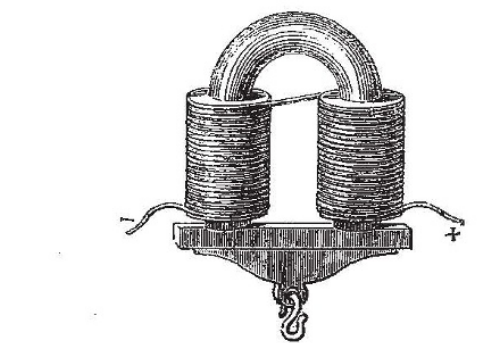

Fig. I4,-Horse-shoe electro-magnet, with armatuxe.

piece of soft iron, and an electric current is passed through the wire helix so wound round the soft iron core (Fig. I4), the soft iron becomes a magnet and remains so, so long as the current flows through the wire; when the current ceases, the soft iron is no longer a magnet; the polarity of this magnet is reversed according to the direction in which the current is sent through the coil or the direction in which the wire is wound round the soft iron core.

When a coil of wire surrounding a soft iron core is passed before the pole of a permanent-magnet, at the moment of passing it becomes a magnet by induction, and at the instant of making and breaking contact with the pole of the permanent-magnet, a wave of magnetoelectricity is induced in the coil of wire surrounding the soft iron core. The current induced at the breaking is in an opposite direction and stronger than the current induced at the making contact. The more rapid and decided the make and break, the stronger the magneto. currents induced in the coil.

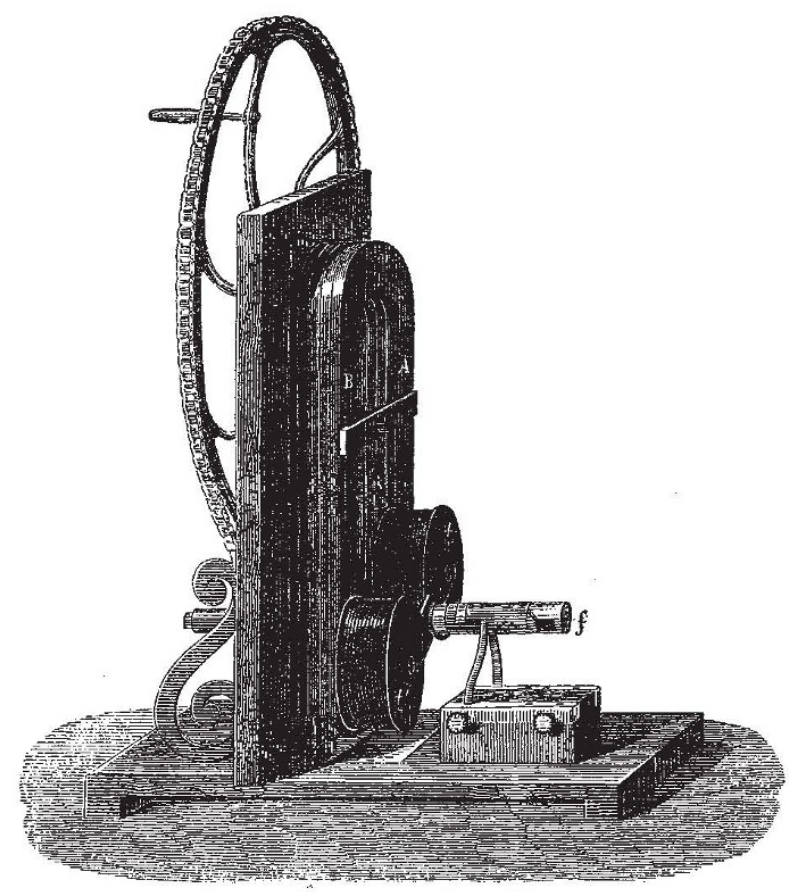

Frg. 15-Magneto-electric machine.

A magneto-machine of this description is shown in Fig. 15. It consists of a powerful permanent magnet, A, B, composed of steel plates in the form of a horse-shoe, firmly fixed in a vertical position to a wooden frame, the two poles of the magnet being opposite to two coils of insulated wire, each furnished with a soft iron core. These two soft iron cores are connected together by an iron plate, $t, t^{\prime}$; the coils thus arranged constitute an electro-magnet. The electro-magnet thus formed is fixed so as to revolve round an axis, $f$, which passes between the poles of the magnet, and is connected with an endless chain and wheel with a handle.

When the coils are put in motion, induced currents of magneto-electricity are developed in each of them, at each successive make and break of the soft iron cores with the poles of the magnet $\mathrm{A}, \mathrm{B}$. If the wires of the coils are wound in contrary directions, the induced currents developed in each coil by the approach of the two contrary poles of the magnet will be in the same direction.

When insulated wire helices are placed round the two poles of a permanent-magnet, so that a continuous circuit is formed, and an armature of soft iron is rotated before them, at the moments of the make and break of the revolving armature with the poles of the magnet, a wave of magneto-electricity is induced in the wire helices, the stronger current being that produced by the breaking contact, which is in an opposite direction to the weaker current induced in the helices at the moment of making contact. 
When the poles of two permanent-magnets are opposed to each other, the similar poles will exert a repellant, and the dissimilar poles an attractive force. This principle is constant, whether the magnets are electro-magnets or permanent-magnets.

In a permanent-magnet, as is well understood, the magnetic force culminates at the two opposite extremities of the bar, and for the purposes of telegraphy may be considered as equivalent to the force emanating from the two poles of a voltaic series, but more lasting ; there is no battery to be renewed, the excitation of the current is mechanical, and not chemical.

When a piece of soft iron is placed close to the poles of a permanent-magnet (Fig. , $_{5}$ ), it will become a magnet by induction, and the polarity of the ends will be dissimilar in their nature to those of the permanent-magnet.

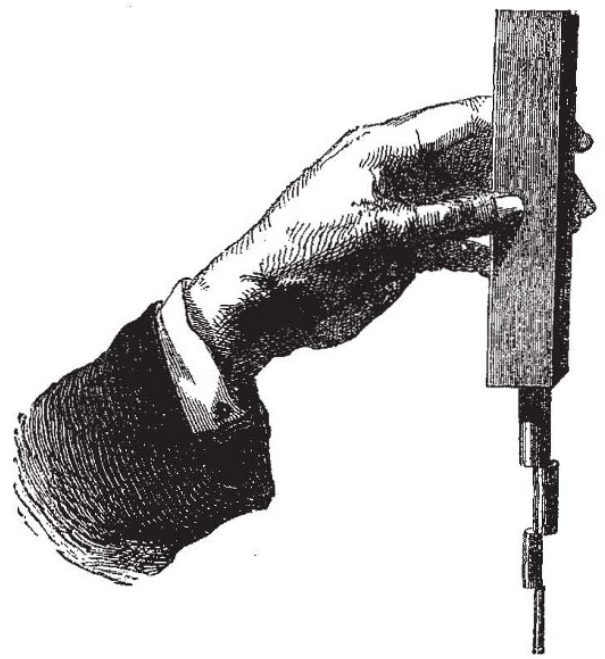

FIG. 16.-Magnetisation of pieces of soft iron by the influence of magnetism (induction).

When the pole of a permanent-magnet is placed within a hollow coil or helix of insulated wire freely suspended so as to oscillate on an axis, and a current of electricity is passed through the helix, it will be oscillated or rotated towards the right or left over the poles of the magnet according to the direction of the current.

In a similar way, when a permanent magnetic bar is freely suspended within a hollow coil or helix of wire, the magnetic bar will oscillate to the right or left, according to the direction in which the current flows through the helix.

These are the principal fundamental laws which, combined together in various mechanical details, constitute every form of telegraphic apparatus known; and it is upon the accurate balance of the resistances, and delicacy of the mechanical parts, that the excellence of the instrument for practical purposes depends. It will now be pointed out how these well-known principles have been combined to produce the beautiful machines at present employed upon submarine circuits of extended length, and by which, with feeble currents, signals are automatically recorded at the distant station.

Commencing with non-recording instruments, the mirror galvanometer is at once the most useful and important in its general applications to submarine telegraphy. The electrical combinations of principles which constitute this instrument existed almost in the same arrangement in the earliest days of telegraphic research. At that early period the apparatus in its elementary conditions was almost identical with the modern instrument, the baralmost identical with thed in the centre of a hollow coil of insulated wire, and the focal distance at which to observe the angular motion of the suspended needle to the right or left. In this crude arrangement there existed the germ of the instrument now in use, the accurate balance of resistances, and delicate adjustments of the mechanical parts, producing the difference between an historical invention and an every-daypractical mechanical application. The construction of the reflecting galvanometer is exceedingly simple, the delicacy of the instrument being the result of the lightness of the moving parts.

Two hollow coils of fine wire (Fig. I7), united to form a continuous circuit, are placed one above the other, and the coils are so constructed as to admit of a very delicate axis being inserted through them free to rotate and capable of accurate adjustment, so that the centre of rotation may be in a line with the centre of the inner ring of the coils. A minute silvered mirror reflector is attached to the axis concentric with the hollow centre of the upper coil. Two extremely light bar-magnets, about three-eighths of an inch in length, are attached to the axis in the centre of each coil, one of the magnets being therefore at the back of the mirror. The polarity of these bar-magnets is reversed, producing an astatic combination. The whole arrangement of axis, mirror, and bar-magnets is suspended by a cocoon fibre, ajjustments being obtained to ensure freedom of rotation by a micrometer screw and levelling screws. The mirror is brought into the field and its motion otherwise controlled by means of a permanent-magnet sliding upon the rod, the elevation or depression of which acting by induction upon the suspended bar-magnets gives more or less sensitiveness to the motion of the mirror when a current of electricity traverses the coils. It must be obvious that the eye is quite incapable of detecting with accuracy the minute angular motions of the mirror, and that some means must be employed to magnify and increase this angular motion of the magnetic bar. For this purpose a beam of light is employed which, falling on the mirror, is reflected back again upon a long horizontal scale placed some six feet off. The angle of incidence of the beam of light being equal to the angle of reflection, the oscillation of the mirror thus magnified to the eye, to right or left, is read off from a zero on the scale. The beam of light is passed through an adjusting slit immediately beneath the scale, and the mirror is brought to the zero of the scale by the magnetic adjustment before mentioned. Thus the slightest angular motion of the mirror, inappreciable to the eye, is, according to the focal length of the ray of light, increased to such an extent as to indicate the presence of the most feeble currents with an almost inappreciable movement of the mirror.

The scientific world is indebted to Prof. Sir William Thomson for this exceedingly beautiful adaptation and combination of existing laws, parts, and principles; the skilful balance of which has resulted in an apparatus now almost exclusively used for the testing of the electric condition of submarine cables. It is obvious that with this reflecting galvanometer no automatic register of the signals received can be obtained; recourse is therefore had to a Morse key, by means of which the recipient of the signal at once records the deflection of the light spot on the scale to the right or left in the symbolic Morse code of the dot and dash. The mirror galvanometer, in fact, occupies relatively the same position in electrical mechanics as the violin dces in musical acoustics. In the violin, by sliding the finger up the string and thus shortening the length of the vibrating string, the musical pitch or tone produced from the string, as the bow is drawn across it, continues to ascend in the musical scale without break; each note of the entire diatonic scale capable of being produced on that string, sliding or melting into the next, the pitch of the note being the index or record of the length of the string and numerical value of its vibrations.

In a similar manner the great peculiarity of the mirror or reflecting galvanometer is, that it continuously 
indicates and measures with great exactitude the various increases in the power or strength of the received electric current, the motion of the spot of light following every variation of the current, thus affording a record to the eye of the value of the current under circumstances and conditions in which ordinary instruments indicating the mere presence or absence of a given strength of current would be valueless.

In the Syphon Recorder about to be brought under notice, the same remarkable feature of presenting the ever-varying value of the received current at the end of

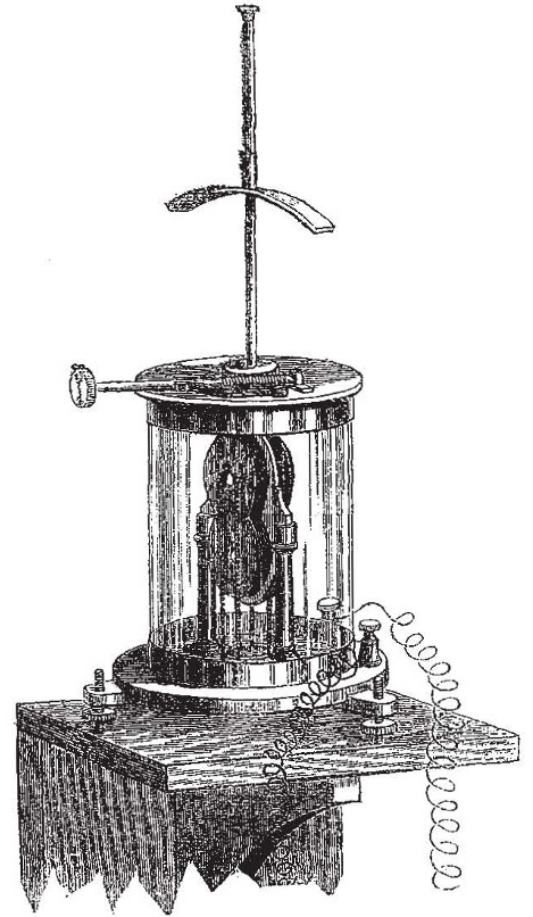

FIG. I7.-Sir William Thomson's "mirror" or reflecting galvanometer.

he has combined well-keown forms and principles together in such an arrangement and combination of parts as to produce new and useful results. It is combinations such as those now to be described that constitute the value of scientific research in relation to mechanical applications.

It should be remembered that it is not possible to patent a principle, but only the application of a principle. If this axiom were more frequently remembered, the severe strictures upon patents in general that have already the cable, or the strength of the current, is preserved while at the same time a permanent automatic record is registered for reference.

The difficulty to be overcome in the construction of such a recording instrument has been chiefly that due to the mechanical problem of obtaining marks from a very light body in rapid motion without impeding or interfering with that motion. The combination of parts and principles employed in the syphon recorder will be found to be more or less previously well known; the merit of Sir William Thomson's beautiful instrument consists in that

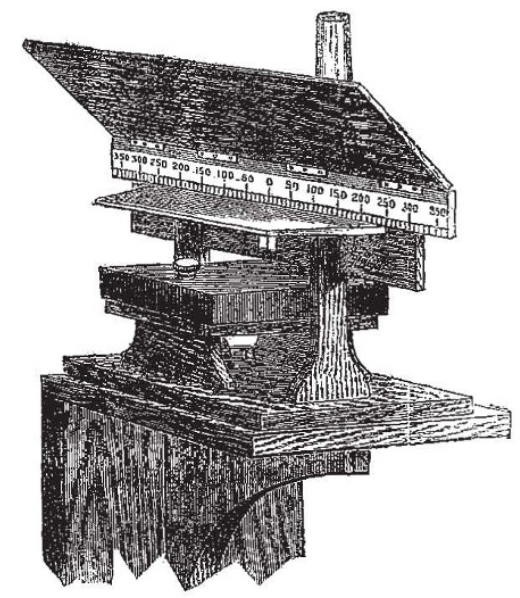

been made in these articles would have been rendered unnecessary. The "syphon recording" instrument fitly illustrates in what a good and valuable patent consists. With old and well-known parts and principles such as permanent-magnets, electro-magnets, coils, armatures, syphons, capillary attraction, and hydrostatic pressure and such like material, novel and practical results have been produced.

\section{(To be continuied.)}

\section{OUR ASTEONONICAL COLUMN}

THE SOlar ECLIPSE OF I900, May 28.-We refer to this eclipse with the view of correcting an error in Hallaschka's "Elementa Eclipsium," where it is stated to be annular. It is really the last total eclipse visible in Europe during the present century. The following elements may be expected to be pretty near the true ones:-

Conjunction in R.A. May 28, at 2h. $56 \mathrm{~m}, 22 \mathrm{~s}$. G.M.T.

R.A.

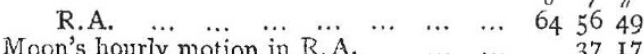

$\begin{array}{lllll}\text { Sun's } \quad, \quad & \ldots & \ldots & 2 & 22\end{array}$

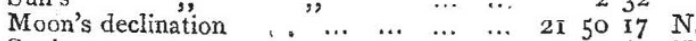

$\begin{array}{lllllllll}\text { Sun's } \quad, n & , & \ldots & \ldots & 21 & 27 & \text { is } & \mathrm{N}\end{array}$
Moon's hourly motion in Decl.

Sun's

Moon's horizontal parallax"

Sun's , ", semidiameter

Sun's$$
\text { , }
$$

\begin{tabular}{|c|c|c|}
\hline$\ldots$ & $\cdots$ & $2^{\prime} \quad 39$ \\
\hline$\ldots$ & $\ldots$ & 024 \\
\hline$\ldots$ & $\ldots$ & $\begin{array}{lll}58 & 27\end{array}$ \\
\hline$\ldots$ & $\ldots$ & ० 9 \\
\hline$\ldots$ & $\ldots$ & 1556 \\
\hline$\ldots$ & $\ldots$ & r5 4 \\
\hline
\end{tabular}

The sidereal time at Greenwich mean noon, is 4h. $22 \mathrm{~m}$. $16 \mathrm{~s}^{\prime} 8$, and the equation of time $2 \mathrm{~m} .59 \mathrm{~s}$. additive to mean time.

The central eclipse enters Europe near Ovar, on the coast of Portugal, and passes off Spain a little south of Alicante. In longitude oh. $34 \mathrm{~m}$. Os. W. and latitude $40^{\circ} 49^{\prime} \mathrm{N}$. on the Portuguese coast, totality commences at $3 \mathrm{~h} .27 \mathrm{~m}$. local mean time, and continues Im. 30s., the sun at an altitude of about $43^{\circ}$. At Alicante it commences at $4 \mathrm{~h}$. Iom. IIS. 